\title{
The use of Groups, Algorithms and Programming (GAP) software in calculating the commutator subgroup and centre of groups of order $p^{3} q$
}

\author{
Samad Rashid ${ }^{a, *}$, Adnin Afifi Nawi ${ }^{\mathrm{b}}$, Rosita Zainal ${ }^{\mathrm{b}}$, Nor Muhainiah Mohd Ali ${ }^{\mathrm{b}}$, Nor Haniza Sarmin ${ }^{\mathrm{b}}$ \\ ${ }^{a}$ Department of Mathematics, College of Basic Science, Yadegar-e-Imam Khomeini (RAH) Branch, \\ Islamic Azad University, Tehran, Iran \\ b Department of Mathematical Sciences, Faculty of Science, Universiti Teknologi Malaysia, \\ 81310 UTM Johor Bahru, Johor, Malaysia
}

*Corresponding author, e-mail: samadrashid47@yahoo.com

Received 13 Feb 2014

Accepted 9 Jun 2014

\begin{abstract}
In this study, we determine the commutator subgroup and centre of groups of order $p^{3} q$, where $p$ and $q$ are distinct primes and $p<q$. The software package Groups, Algorithms and Programming (GAP) is used to verify the hand calculation of the commutator subgroup and centre of this group. From the results obtained, the commutator subgroup and centre of the groups are partitioned into some cases depending on their classification.
\end{abstract}

KEYWORDS: finite groups, finitely generated groups, derived subgroup

\section{INTRODUCTION}

Let $G$ be a group. The commutator subgroup of $G$, which will be denoted by $G^{\prime}$, is a subgroup of $G$ generated by all commutators in $G$. Meanwhile, the centre of a group $G, Z(G)$, is the set of elements in $G$ that commute with every element of $G$. Consider the homomorphism $\tau G \rightarrow$ Aut $G$ defined by $(x) g^{\tau}=x^{g}$ with image $\operatorname{Inn} G$. The kernel of $\tau$ is precisely the centre, $Z(G)^{1}$.

The commutator subgroup and centre of the group are important elements in determining the non-abelian tensor square, the Schur multiplier and capability of groups. The Schur multiplier, non-abelian tensor square, and capability of groups of order $p^{2} q$, where $p$ and $q$ are distinct primes, have been determined by Rashid et $\mathrm{al}^{2}$. Meanwhile, Rashid et $\mathrm{al}^{3}$ determined the non-abelian tensor square for groups of order $8 q$ where $q$ is an odd prime. Zainal et $\mathrm{al}^{4}$ focused on the non-abelian tensor square of non-abelian group of order $p^{4}$, where $p$ is an odd prime. Groups, Algorithms and Programming (GAP) software ${ }^{5}$ is a system for computational discrete algebra, with particular emphasis on Computational Group Theory. GAP also provides large amounts of data quickly so that these data can be used to formulate a conjecture. In some cases, by the use of GAP, the produced patterns can be used to approach for proving a theorem. In this study, we use GAP software to compute the commutator subgroup and centre of groups of order $p^{3} q$ where $p$ and $q$ are distinct prime for some small $p$ and $q$ and $p<q$. The results are then proved in general.

\section{PRELIMINARIES}

This section includes some basic results which are necessary in this study.

In 1899, the classification of groups of order $p^{3} q$ has been obtained by Western ${ }^{6}$. He proved that there are 27 types of groups of order $p^{3} q$, where $p<q$. The classification of groups of order $p^{3} q$, where $p<q$ is stated in the following theorem.

Theorem 1 [Western] Let $G$ be a non-abelian group of order $p^{3} q$, where $p$ and $q$ are distinct prime and $p<q$. Then exactly one of the following holds:

$$
\begin{gathered}
G_{1} \cong\langle a, b, c| a^{4}=b^{2}=c^{q}=1, b a b=a^{-1}, a c=c a, \\
b c=c b\rangle \\
G_{2} \cong\langle a, b, c| a^{4}=b^{4}=c^{q}=1, b^{2}=a^{2}, \\
\left.b^{-1} a b=a^{-1}, a c=c a, b c=c b\right\rangle \\
G_{3} \cong\left\langle a, b \mid a^{8}=b^{q}=1, a^{-1} b a=b^{-1}\right\rangle \\
G_{4} \cong\langle a, b, c| a^{4}=b^{2}=c^{q}=1, a b=b a, a c=c a, \\
\left.b c b=c^{-1}\right\rangle \\
G_{5} \cong\langle a, b, c| a^{4}=b^{2}=c^{q}=1, a b=b a, \\
\left.a^{-1} c a=c^{-1}, b c=c b\right\rangle
\end{gathered}
$$




$$
\begin{aligned}
& G_{6} \cong\langle a, b, c| a^{4}=b^{2}=c r=d^{q}=1, \\
& a b=b a, a c=c a, b c=c b, \\
& \left.a d=d a, b c=c b, c d c=d^{-1}\right\rangle \\
& G_{7} \cong\langle a, b, c| a^{4}=b^{2}=c^{q}=1, b a b=a^{-1} \text {, } \\
& \left.a c=c a, b c b=c^{-1}\right\rangle \\
& G_{8} \cong\langle a, b, c| a^{4}=b^{2}=c^{q}=1, b a b=a^{-1} \text {, } \\
& \left.a^{-1} c a=c^{-1}, b c=c b\right\rangle \\
& \text { where } q \equiv 1(\bmod 2) \\
& G_{9} \cong\langle a, b, c| a^{4}=b^{4}=c^{q}=1, b^{2}=a^{2}, \\
& b^{-1} a b=a^{-1}, a c=c a, \\
& \left.b^{-1} c b=c^{-1}\right\rangle \\
& G_{10} \cong\left\langle a, b, c \mid a^{8}=b^{q}=1, a^{-1} b a=c^{m}\right\rangle \\
& \text { where } m \text { is any primitive root of } \\
& m^{4} \equiv 1(\bmod q) \text { and } q \equiv 1(\bmod 4) . \\
& G_{11} \cong\langle a, b, c| a^{4}=b^{2}=c^{q}=1, a b=b a, \\
& \left.a^{-1} c a=c^{m}, b c=c b\right\rangle \\
& \text { where } m \text { is any primitive root of } \\
& m^{8} \equiv 1(\bmod q) \text { and } q \equiv 1(\bmod 8) \text {. } \\
& G_{12} \cong\left\langle a, b \mid a^{8}=b^{q}=1, a^{-1} b a=b^{m}\right\rangle \\
& \text { where } m \text { is any primitive root of } \\
& m^{8} \equiv 1(\bmod q) \text { and } q \equiv 1(\bmod 8) \text {. } \\
& G_{13} \cong\langle a, b, c, d| a^{2}=b^{2}=c^{2}=d^{q}=1, a b=b a, \\
& a c=c a, b c=c b, a d=d a, \\
& \left.d^{-1} b d=c, d^{-1} c d=b c\right\rangle \\
& G_{14} \cong\langle a, b, c| a^{4}=b^{4}=c^{3}=1, a^{2}=b^{2} \text {, } \\
& b^{-1} a b=a^{-1}, c^{-1} a c=b, \\
& \left.c^{-1} b c=a c\right\rangle \\
& G_{15} \cong\langle a, b, c| a^{4}=b^{4}=c^{3}=1, b a b=a^{-1}, \\
& c^{-1} a^{2} b=b, c^{-1} b c=a^{2} b, \\
& \left.a^{-1} c a=c^{2} a^{2} b\right\rangle \\
& G_{16} \cong\langle a, b, c, d| a^{2}=b^{2}=c^{2}=d^{7}=1, a b=b a, \\
& a c=c a, b c=c b, d^{-1} a d=b, \\
& \left.d^{-1} b d=c, d^{-1} c d=a b\right\rangle \\
& G_{17} \cong\langle a, b, c| a^{p^{2}}=b^{p}=c^{q}=1, b^{-1} a b=a^{p+1}, \\
& a c=c a, b c=c b\rangle \\
& G_{18} \cong\langle a, b, c, d| a^{p}=b^{p}=c^{p}=d^{q}=1, a b=b a, \\
& a c=c a, c^{-1} b c=a b, a d=d a, \\
& b d=d b, c d=d c\rangle \\
& G_{19} \cong\left\langle a, b \mid a^{p^{3}}=b^{q}=1, a^{-1} b a=b^{m} a\right\rangle
\end{aligned}
$$

where $m$ is any primitive root $[0,1]$

of $m^{p} \equiv 1(\bmod q)$ and $q \equiv 1(\bmod p)$.

$$
G_{20} \cong\langle a, b, c| a^{p^{2}}=b^{p}=c^{q}=1, a b=b a,
$$$$
\left.a c=c a, b^{-1} c b=c^{m}\right\rangle
$$

where $m$ is any primitive root of $m^{p} \equiv 1(\bmod q)$ and $q \equiv 1(\bmod p)$.

$$
\begin{gathered}
G_{21} \cong\langle a, b, c, d| a^{p}=b^{p}=c^{p}=d^{q}=1, a b=b a, \\
a c=c b, a d=d a, b d=d b, \\
\left.c^{-1} d c=d^{m}\right\rangle
\end{gathered}
$$

where $m$ is any primitive root of $m^{p} \equiv 1(\bmod q)$ and $q \equiv 1(\bmod p)$.

$$
\begin{gathered}
G_{22} \cong\langle a, b, c| a^{p^{2}}=b^{p}=c^{q}=1, b^{-1} a b=a^{p+1}, \\
\left.a c=c a, b^{-1} c b=Q^{n}\right\rangle \\
\text { where } m \text { is any primitive root of } \\
m^{p} \equiv 1(\bmod q) \text { and } q \equiv 1(\bmod p) \\
\text { and } n=m, m^{2}, \ldots, m^{p-1} . \\
G_{23} \cong\langle a, b, c, d| a^{p}=b^{p}=c^{p}=d^{q}=1, a b=b a, \\
a c=c b, \text { ad }=d a, b d=d b, \\
\left.c^{-1} b c=a b, c^{-1} d c=d^{m}\right\rangle \\
\text { where } m \text { is any primitive root of } \\
m^{p} \equiv 1(\bmod q) \text { and } q \equiv 1(\bmod p) . \\
G_{24} \cong\left\langle a, b \mid a^{p^{3}}=b^{q}=1, a^{-1} b a=b^{m}\right\rangle \\
\text { where } m \text { is any primitive root of } \\
m^{p^{2}} \equiv 1(\bmod q) \text { and } q \equiv 1\left(\bmod p^{2}\right) . \\
m^{p^{2}} \equiv 1(\bmod q) \text { and } q \equiv 1\left(\bmod p^{3}\right) . \\
G_{25} \cong\left\langle a, b \mid a^{p^{3}}=b^{q}=1, a^{-1} b a=b^{m}\right\rangle \\
\text { where } m \text { is any primitive root of } \\
m^{p^{2}} \equiv 1(\bmod q) \text { and } q \equiv 1\left(\bmod p^{2}\right) . \\
\text { ab } \left.=b a, a^{-1} \text { ca }=c^{a}, b c=c b\right\rangle \\
\text { where } m \text { is any primitive root of }
\end{gathered}
$$

\section{GAP COMPUTATIONS}

From Theorem 1, we can see that there are groups of order $p^{3} q$, with 2,3 , or 4 generators. Since the computation of the commutator subgroup and centre for all 2, 3, or 4-generator groups is similar, the commutator subgroup and centre are computed in details for exactly one type of group from each family of generators stated earlier.

In the first step, we use GAP to compute the commutator subgroup of groups of order $p^{3} q$. 
GAP was used to compute the commutator subgroup for groups of type $G_{1}, G_{3}$ and $G_{6}$ with $q=3$ and presented as follows:

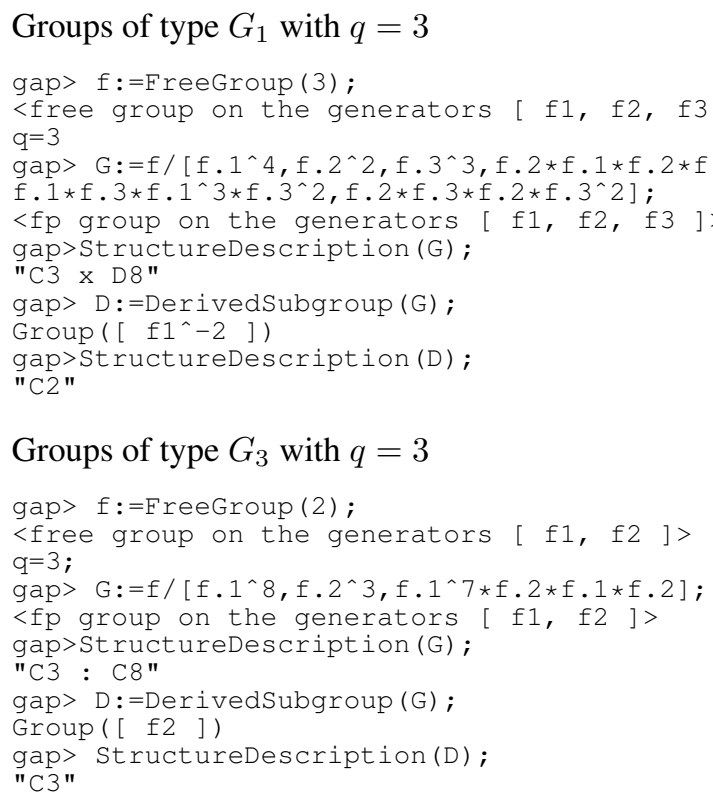

Groups of type $G_{6}$ with $q=3$

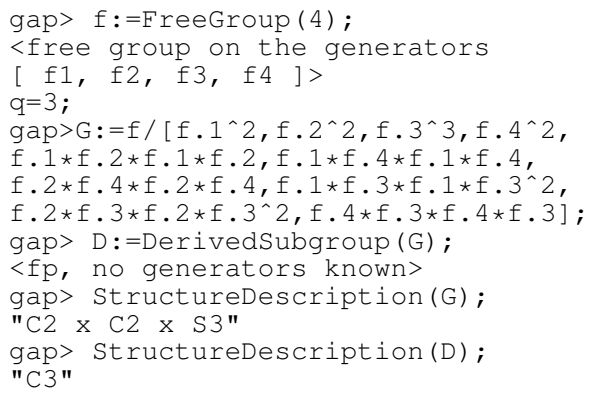

These computations show that the commutator subgroup for groups of type $G_{1}$ with $q=3$ is a cyclic group of order 2 which is isomorphic to $\mathbb{Z}_{2}$ while the commutator subgroup for groups of type $G_{3}$ and $G_{6}$ with $q=3$ is a cyclic group of order 3 which is isomorphic to $\mathbb{Z}_{3}$.

Next, the GAP programmes which are used to compute the centre for groups of type $G_{1}$ and $G_{3}$ with $q=3,5$, and 7 and groups of type $G_{6}$ with $q=11$ are shown below.

Groups of type $G_{1}$ with

$q=3$

gap> f:=FreeGroup (3) i

<free group on the generators [f1, f2, fl] > gap> G: $=\mathrm{f} /\left[\mathrm{f} .1^{\wedge} 4, \mathrm{f} .2^{\wedge} 2, \mathrm{f} .3^{\wedge} 3, \mathrm{f} .2 * \mathrm{f} .1 * \mathrm{f} .2 * \mathrm{f} .1\right.$, f. $1 * \mathrm{f} .3 * \mathrm{f} .1 \wedge 3 * \mathrm{f} .3^{\wedge} 2$, f. $\left.2 * \mathrm{f} .3 * \mathrm{f} .2 * \mathrm{f} .3^{\wedge} 2\right]$;

$<\mathrm{fp}$ group on the generators $[\mathrm{f} 1, \mathrm{f} 2, \mathrm{f} 3]>$

gap> C:=Center $(\mathrm{G})$;

Group ( [ f $3, \mathrm{f} 2 * \mathrm{f} 1 * \mathrm{f} 2^{\wedge}-1 * \mathrm{f} 1^{\wedge}-1, \mathrm{f} 2 \wedge 2$,

$\mathrm{f} 2 * \mathrm{f} 3 * \mathrm{f} 2^{\wedge}-1, \mathrm{f} 1^{\wedge} 2, \mathrm{f} 1 * \mathrm{f} 3 * \mathrm{f} 1^{\wedge}-1$, $\mathrm{f} 1 * \mathrm{f} 2 * \mathrm{f} 1 * \mathrm{f} 2^{\wedge}-1, \mathrm{f} 1 * \mathrm{f} 2^{\wedge} 2 * \mathrm{f} 1^{\wedge}-1$

$\left.\mathrm{f} 1 * \mathrm{f} 2 * \mathrm{f} 3 * \mathrm{f} 2^{\wedge}-1 * \mathrm{f} 1^{\wedge}-1\right]$ )

gap> StructureDescription (C) ;

$q=5$

gap> f:=FreeGroup (3) ;

<fp group on the generators [ $f 1, f 2, f 3]>$

gap> G: $=\mathrm{f} /\left[\mathrm{f} .1^{\wedge} 4, \mathrm{f} .2^{\wedge} 2, \mathrm{f} .3^{\wedge} 5, \mathrm{f} .2 * \mathrm{f} .1 * \mathrm{f} .2 * \mathrm{f} .1\right.$,

f. $\left.1 * \mathrm{f} .3 * \mathrm{f} .1 \wedge 3 * \mathrm{f} .3^{\wedge} 4, \mathrm{f} .2 * \mathrm{f} .3 * \mathrm{f} .2 * \mathrm{f} .3^{\wedge} 4\right]$;

gap> C:=Center $(\mathrm{G})$;

Group ( [ f $3, f 2 * f 1 * f 2^{\wedge}-1 * f 1^{\wedge}-1, f 2^{\wedge} 2$,

$\mathrm{f} 2 * \mathrm{f} 3 * \mathrm{f} 2^{\wedge}-1, \mathrm{f} 1^{\wedge} 2, \mathrm{f} 1 * \mathrm{f} 3 * \mathrm{f} 1^{\wedge}-1$

$\mathrm{f} 1 * \mathrm{f} 2 * \mathrm{f} 1 * \mathrm{f} 2^{\wedge}-1, \mathrm{f} 1 * \mathrm{f} 2 \wedge 2 * \mathrm{f} 1^{\wedge}-1$,

$\left.\mathrm{f} 1 * \mathrm{f} 2 * \mathrm{f} 3 * \mathrm{f} 2^{\wedge}-1 * \mathrm{f} 1^{\wedge}-1\right]$ )

gap> StructureDescription (C) ;

"C10"

$q=7$

gap>f:=FreeGroup (3) ;

<fp group on the generators [f1, f2, f3] $>$ gap> G: $=\mathrm{f} /\left[\mathrm{f} .1^{\wedge} 4, \mathrm{f} .2^{\wedge} 2, \mathrm{f} .3^{\wedge} 7, \mathrm{f} .2 * \mathrm{f} .1 * \mathrm{f} .2 * \mathrm{f} .1\right.$, f. $\left.1 * \mathrm{f} .3 * \mathrm{f} .1^{\wedge} 3 * \mathrm{f} .3^{\wedge} 6, \mathrm{f} .2 * \mathrm{f} .3 * \mathrm{f} .2 * \mathrm{f} .3^{\wedge} 6\right]$

gap> C:=Center $(\mathrm{G})$;

Group [f3, f $2 * \mathrm{f} 1 * \mathrm{f} 2^{\wedge}-1 * \mathrm{f} 1^{\wedge}-1, \mathrm{f} 2^{\wedge} 2, \mathrm{f} 2 * \mathrm{f} 3 * \mathrm{f} 2^{\wedge}-1$, $\mathrm{f} 1 \wedge 2, \mathrm{f} 1 * \mathrm{f} 3 * \mathrm{f} 1^{\wedge}-1, \mathrm{f} 1 * \mathrm{f} 2 * \mathrm{f} 1 * \mathrm{f} 2^{\wedge}-1, \mathrm{f} 1 * \mathrm{f} 2 \wedge 2 * \mathrm{f} 1^{\wedge}-1$, $\left.\mathrm{f} 1 * \mathrm{f} 2 * \mathrm{f} 3 * \mathrm{f} 2^{\wedge}-1 * \mathrm{f} 1^{\wedge}-1\right]$ )

gap> StructureDescription (C) ;

"C1 4 "

\section{Groups of type $G_{3}$ with}

$q=3$

gap> f:=FreeGroup (2)

<free group on the generators [f1, f2 ]>

gap> G:=f/[f.1^8,f.2^3,f.1^7*f.2*f.1*f.2];

gap $>C:=$ Center $(G)$

Group ( [f $2^{\wedge} 2 * \mathrm{f} 1^{\wedge}-1 * \mathrm{f} 2^{\wedge}-1 * \mathrm{f} 1^{\wedge}-1, \mathrm{f} 2 * \mathrm{f} 1^{\wedge} 2 * \mathrm{f} 2^{\wedge}-1$,

$\mathrm{f} 2 * \mathrm{f} 1 * \mathrm{f} 2 * \mathrm{f} 1^{\wedge}-1, \mathrm{ff} 1^{\wedge} 2, \mathrm{f} 1 * \mathrm{f} 2^{\wedge} 2 * \mathrm{f} 1^{\wedge}-1 * \mathrm{f} 2^{\wedge}-1$,

$\left.\left.\mathrm{f} 1 * \mathrm{f} 2 * \mathrm{f} 1 \wedge 2 * \mathrm{f} 2^{\wedge}-1 * \mathrm{f} 1^{\wedge}-1, \mathrm{f} 1 * \mathrm{f} 2 * \mathrm{f} 1 * \mathrm{f} 2\right]\right)$

gap> StructureDescription (C) ;

"C4"

$q=5$

gap> f:=FreeGroup (2) ;

<free group on the generators [f1, f2 ]>

gap $>\mathrm{G}:=\mathrm{f} /\left[\mathrm{f} .1^{\wedge} 8, \mathrm{f} . \mathrm{2}^{\wedge} 5, \mathrm{f} .1^{\wedge} 7 * \mathrm{f} .2 * \mathrm{f} .1 * \mathrm{f} .2\right]$;

gap $>C:=$ Center $(\mathrm{G})$;

Group ( [f $2 * f 1^{\wedge} 2 * f 2^{\wedge}-1, f 2 * f 1 * f 2 * f 1^{\wedge}-1, f 2^{\wedge} 3 * f 1^{\wedge}-$

$1 * \mathrm{f} 2^{\wedge}-2 * \mathrm{f} 1^{\wedge}-1, \mathrm{f} 2^{\wedge} 2 * \mathrm{f} 1^{\wedge} 2 * \mathrm{f} 2^{\wedge}-2, \mathrm{f} 2^{\wedge} 2 * \mathrm{f} 1 * \mathrm{f} 2 * \mathrm{f} 1^{\wedge}-$

$1 * \mathrm{f} 2^{\wedge}-1, \mathrm{f} 1 \wedge 2, \mathrm{f} 1 * \mathrm{f} 2 * \mathrm{f} 1 \wedge 2 * \mathrm{f} 2^{\wedge}-1 * \mathrm{f} 1^{\wedge}-1$,

$\mathrm{f} 1 * \mathrm{f} 2 * \mathrm{f} 1 * \mathrm{f} 2, \mathrm{f} 1 * \mathrm{f} 2^{\wedge} 3 * \mathrm{f} 1^{\wedge}-1 * \mathrm{f} 2^{\wedge}-2$,

$f 1 * f 2^{\wedge} 2 * f 1^{\wedge} 2 * f 2^{\wedge}-2 * f 1^{\wedge}-1$

$\left.\left.\mathrm{f} 1 * \mathrm{f} 2 \wedge 2 * \mathrm{f} 1 * \mathrm{f} 2 * \mathrm{f} 1^{\wedge}-1 * \mathrm{f} 2^{\wedge}-1 * \mathrm{f} 1^{\wedge}-1\right]\right)$

gap> StructureDescription $(\mathrm{C})$;

$q=7$

gap> f:=FreeGroup (2)

<free group on the generators [f1, f2 ]>

gap> G:=f/[f.1^8,f.2^7,f.1^7*f.2*f.1*f.2]

gap> C:=Center $(G)$;

Group ( $<15$ generators $>$ )

gap> StructureDescription (C) ;

"C4 "

Groups of type $G_{6}$ with $q=11$

<fp group on the generators $[\mathrm{f} 1, \mathrm{f} 2, \mathrm{f} 3, \mathrm{f} 4]>$

gap $>\mathrm{G}:=\mathrm{f} /\left[\mathrm{f} .1^{\wedge} 2, \mathrm{f} .2^{\wedge} 2, \mathrm{f} .3^{\wedge} 7, \mathrm{f} .4^{\wedge} 2\right.$,

f. $1 * \mathrm{f} .2 * \mathrm{f} .1 * \mathrm{f} .2$, f. $1 * \mathrm{f} .4 * \mathrm{f} .1 * \mathrm{f} .4$,

f. $2 * f .4 * f .2 * f .4, f .1 * f .3 * f .1 * f .3^{\wedge} 10$ 
f. $\left.2 * f .3 * f .2 * f .3^{\wedge} 10, f .4 * f, 3 * f, 4 * f, 3\right]$;

gap> C:=Center $(\mathrm{G})$;

Group (<67 generators $>$ )

gap> StructureDescription(C);

"C2 × C2"

The above computations show that the centre for groups of type $G_{1}$ with $q=3,5$, and 7 are cyclic groups of order 6,10 , and 14 , respectively, while the centre for groups of type $G_{3}$ with $q=3,5$, and 7 are cyclic groups of order 4 and the centre for groups of type $G_{6}$ with $q=11$ are isomorphic to $\mathbb{Z}_{2} \times \mathbb{Z}_{2}$.

Consider the pattern given by GAP algorithms, we obtained Theorem 2 and Theorem 3. We also provide the proofs for certain type of groups in the classification.

\section{MAIN RESULTS}

In this section, we presented our results in general. The first theorem gives the commutator subgroup for non-abelian group of order $p^{3} q$ while the second theorem gives the centre of all the groups in the classification.

Theorem 2 Let $G$ be a non-abelian group of order $p^{3} q$, where $p$ and $q$ are distinct prime and $p<q$. Then the commutator subgroup of $G$ is exactly one of the following:

$G^{\prime} \cong \begin{cases}\mathbb{Z}_{2} ; & G \text { is } G_{1}, G_{2} \\ \mathbb{Z}_{q} ; & G \text { is } G_{3}, G_{4}, G_{5}, G_{6}, G_{10}, G_{11}, G_{12}, \\ \mathbb{Z}_{2 q} ; & G \text { is } G_{7}, G_{8}, G_{9} \\ \mathbb{Z}_{2}^{2} ; & G \text { is } G_{13} \\ Q_{2} ; & G \text { is } G_{14} \\ A_{4} ; & G \text { is } G_{15} \\ \mathbb{Z}_{2}^{3} ; & G \text { is } G_{16} \\ \mathbb{Z}_{p} ; & G \text { is } G_{17}, G_{18} \\ \mathbb{Z}_{q} ; & G \text { is } G_{19}, G_{20}, G_{21}, G_{22}, G_{25}, G_{26} \\ \quad & G_{27}, \\ \mathbb{Z}_{p q} ; & G \text { is } G_{23}, G_{24} .\end{cases}$

Proof: First we prove for $G=G_{1}$. By using the relations $b a b=a^{-1}, a c=a c$ and $b c=c b$, every element of $G$ can be uniquely written in the form $a^{i} b^{j} c^{k}$ where

$$
0 \leqslant i \leqslant 3,0 \leqslant j \leqslant 1 \text { and } 0 \leqslant k \leqslant q-1 .
$$

Suppose that $g=a^{i} b^{j} c^{k}$ and $g^{\prime}=a^{i^{\prime}} b^{j^{\prime}} c^{k^{\prime}}$ be two arbitrary elements of $G$. Then we have

Case 1: $j=j^{\prime}=0$. In this case by the relations, it is clear that $\left[g, g^{\prime}\right]=1$.

Case 2: $j=1, j^{\prime}=0$. By the relations $a c=c a$ and

$$
\begin{aligned}
& b c=c b, \\
& \qquad \begin{aligned}
{\left[g, g^{\prime}\right] } & =a^{i} b c^{k} a^{i^{\prime}} c^{k^{\prime}} c^{-k} b a^{-i} c^{-k^{\prime}} a^{-i^{\prime}} \\
& =a^{i} b c^{k} b a^{-i^{\prime}} c^{k^{\prime}-k} a^{-i} c^{-k^{\prime}} a^{-i^{\prime}} \\
& =a^{-2 i^{\prime}} .
\end{aligned}
\end{aligned}
$$

Case 3: $j=0, j^{\prime}=1$. Similarly, $\left[g, g^{\prime}\right]=a^{-2 i^{\prime}}$.

Case 4: $j=j^{\prime}=1$. In this case,

$$
\begin{aligned}
{\left[g, g^{\prime}\right] } & =a^{i} b c^{k} a^{i^{\prime}} b c^{k^{\prime}} c^{-k} b a^{-i} c^{-k^{\prime}} b a^{-i^{\prime}} \\
& =a^{i} a^{-i^{\prime}} b c^{k} b c^{k^{\prime}-k} b a^{-i} c^{-k^{\prime}} b a^{-i^{\prime}} \\
& =a^{i-i^{\prime}} c^{k+k^{\prime}-k} a^{i} b c^{-k^{\prime}} b a^{-i^{\prime}} \\
& =a^{2\left(i-i^{\prime}\right)} .
\end{aligned}
$$

All these calculations show that $G^{\prime}=\left\{1, a^{2}\right\}$, that is, $G^{\prime} \cong \mathbb{Z}_{2}$.

For determining the commutator subgroup for group $G=G_{3}$, using the relations $a^{-1} b a=b^{-1}$, it is clear that every element of $G$ can be uniquely written in the form $a^{i} b^{j}$ where $0 \leqslant i \leqslant 7$ and $0 \leqslant j \leqslant q-1$. Let $g=a^{i} b^{j}$ and $g^{\prime}=a^{i^{\prime}} b^{j^{\prime}}$ be two arbitrary elements of $G$. Then

$$
\left[g, g^{\prime}\right]=a^{i} b^{j} a^{i^{\prime}} b^{j^{\prime}} b^{-j} a^{-i} b^{-j^{\prime}} a^{-i^{\prime}} .
$$

This calculation shows that

$$
G^{\prime}=\left\{1, b, \ldots, b^{q-1}\right\} \cong \mathbb{Z}_{q} .
$$

For determining the commutator subgroup for group $G=G_{6}$, note that every element of $G$ can be uniquely written in the form $a^{i} b^{j} c^{k} d^{s}$ where $0 \leqslant i, j, k \leqslant 1$ and $0 \leqslant s \leqslant q-1$. Let $g=a^{i} b^{j} c^{k} d^{s}$ and $g^{\prime}=a^{i^{\prime}} b^{j^{\prime}} c^{k^{\prime}} d^{s^{\prime}}$ be two arbitrary elements of $G$. Then

$$
\begin{aligned}
{\left[g, g^{\prime}\right]=a^{i} b^{j} c^{k} d^{s} a^{i^{\prime}} b^{j^{\prime}} c^{k^{\prime}} d^{s^{\prime}} d^{-s} c^{-k} } \\
b^{-j} a^{-i} d^{-s^{\prime}} c^{-k^{\prime}} b^{-j^{\prime}} a^{-i^{\prime}} .
\end{aligned}
$$

The following cases are considered:

Case 1: $k=k^{\prime}=0$. In this case by the relations, it is clear that $\left[g, g^{\prime}\right]=1$.

Case 2: $k=1, k^{\prime}=0$. By the relations,

$$
\begin{array}{r}
{\left[g, g^{\prime}\right]=a^{i} b^{j} c d^{s} a^{i^{\prime}} b^{j^{\prime}} d^{s^{\prime}} d^{-s} c b^{-j}} \\
a^{-i} d^{-s^{\prime}} b^{-j^{\prime}} a^{-i^{\prime}}=d^{-2 s^{\prime}} .
\end{array}
$$

Case 3: $k=0, k^{\prime}=1$. By the similar way, $\left[g, g^{\prime}\right]=$ $d^{-2 s^{\prime}}$.

Case 4: $k=k^{\prime}=1$. In this case $\left[g, g^{\prime}\right]=d^{2\left(s^{\prime}-s\right)}$. Hence we have $G^{\prime}=\left\{1, d, \ldots, d^{q-1}\right\}$, that is, $G^{\prime} \cong \mathbb{Z}_{q}$. 
The proofs of other cases can be done in a similar manner.

In the next theorem, the centre of a non-abelian group of order $p^{3} q$, where $p$ and $q$ are distinct primes and $p<q$ is stated.

Theorem 3 Let $G$ be a non-abelian group of order $p^{3} q$, where $p$ and $q$ are distinct prime and $p<q$. Then exactly one of the following holds:

$$
Z(G) \cong \begin{cases}1 ; & G \text { is } G_{12}, G_{15}, G_{16}, G_{27}, \\ \mathbb{Z}_{2} ; & G \text { is } G_{7}, G_{8}, G_{9}, G_{10}, G_{11}, \\ & G_{13}, G_{14}, \\ \mathbb{Z}_{p} ; & G \text { is } G_{23}, G_{24}, G_{25}, \\ \mathbb{Z}_{2 q} ; & G \text { is } G_{1}, G_{2}, \\ \mathbb{Z}_{q} ; & G \text { is } G_{3}, G_{4}, \\ \mathbb{Z}_{p^{2}} ; & G \text { is } G_{19}, G_{20}, \\ \mathbb{Z}_{p q} ; & G \text { is } G_{17}, G_{18}, \\ \mathbb{Z}_{p} \times \mathbb{Z}_{p} ; & G \text { is } G_{5}, G_{6}, G_{21}, G_{22}, G_{26} .\end{cases}
$$

Proof: There are 27 types of groups in the classification which include 2, 3, or 4 generators of groups. Since the computations of the centre for all 2,3 , or 4-generator groups are similar, $Z(G)$ is computed in details for exactly one type of group from each family of generators stated earlier. In this paper, $Z(G)$ is computed for groups of types $G_{1}, G_{3}$ and $G_{6}$ for 3 , 2 , and 4 generators, respectively.

For computing the centre for the group $G=G_{1}$, let $g=a^{i} b^{j} c^{k}$ be a central element of $G$. Then

$$
a^{i} b^{j} c^{k}=g=g^{a}=a^{i}\left(b^{a}\right)^{j} c^{k}=a^{i} a^{-2 j} b^{j} c^{k},
$$

which implies that $b^{j}=a^{-2 j} b^{j}$, that is $j=0$. Thus $g=a^{i} c^{k}$. On the other hand,

$$
a^{i} c^{k}=g=g^{b}=\left(a^{b}\right)^{i}\left(c^{b}\right)^{k}=a^{-i} c^{k},
$$

it follows that $i=0,2$. Hence, every element of $Z(G)$ can be written in the form of $c^{k}$ or $a^{2} c^{k}$, where

$$
0 \leqslant k \leqslant q-1 .
$$

There exist two types of groups of order $2 q$, namely $\mathbb{Z}_{2 q}$ and $D_{2 q}$ and since in our computation of the centre has an element $a^{2} c$ of order $2 q$, it implies that $Z(G) \cong \mathbb{Z}_{2 q}$.

For the group $G=G_{3}$, let $g=a^{i} b^{j}$ be a central element of $G$. Then

$$
a^{i} b^{j}=g=g^{a}=a^{i}\left(b^{a}\right)^{j}=a^{i} b^{-j},
$$

which implies that $b^{2 j}=1$, when $j=0$. Thus $g=a^{i}$. If $a \in Z(G), a b=b a$. But the relation $b a=a^{-1} b$ implies that $b^{2}=1$, which is a contradiction. By similar way $a, a^{3}, a^{5}, a^{7} \notin Z(G)$, that is,

$$
Z(G)=\left\{1, a^{2}, a^{4}, a^{6}\right\} \cong \mathbb{Z}_{4} .
$$

Subsequently, for the group $G=G_{6}, G \cong D_{q} \times \mathbb{Z}_{2}^{2}$, then

$$
Z(G)=Z\left(\mathbb{Z}_{2} \times \mathbb{Z}_{2}\right) \times Z\left(D_{2 q}\right)=\mathbb{Z}_{2} \times \mathbb{Z}_{2} .
$$

\section{CONCLUSIONS}

In this paper, we have determined the commutator subgroup and centre for all non-abelian groups of order $p^{3} q$ where $p$ and $q$ are distinct prime and $p<q$. The GAP software has been used to facilitate the computation for certain type of groups, in the classification.

\section{REFERENCES}

1. Robinson DJS (1982) A Course in the Theory of Groups. Springer-Verlag, Berlin.

2. Rashid S, Sarmin NH, Erfanian A, Mohd Ali NM (2011) On the non-abelian tensor square and capability of groups of order $p^{2} q$. Arch Math 97, 299-306.

3. Rashid S, Sarmin NH, Erfanian A, Mohd Ali NM, Zainal $\mathrm{R}$ (2013) On the non-abelian tensor square and capability of groups of order 8q. Indagat Math 24, 581-8.

4. Zainal R, Mohd Ali NM, Sarmin NH, Rashid S (2013) On the non-abelian tensor square of groups of order $p^{4}$ where $p$ is an odd prime. Sci Asia 39S, 16-8.

5. The GAP Group (2013) GAP-Groups, Algorithms, and Programming, Version 4.6.3; www.gap-system.org.

6. Western AE (1899) Groups of order $p^{3} q$. Proc Lond Math Soc 30, 209-63. 